\title{
PULSE OXIMETRY WAVE VARIATION AS A NONINVASIVE TOOL TO ASSESS VOLUME STATUS IN CARDIAC SURGERY
}

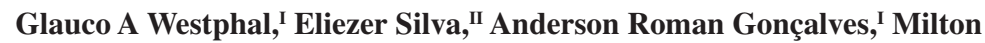 \\ Caldeira Filho, ${ }^{\mathrm{I}}$ Luíz F Poli-de-Figueiredo ${ }^{\mathrm{III}}$ \\ doi: $10.1590 / \mathrm{S} 1807-59322009000400012$
}

Westphal GA, Silva E, Gonçalves AR Caldeira Filho M, Poli-de-Figueiredo LF. Pulse oximetry wave variation as a noninvasive tool to assess volume status in cardiac surgery. Clinics. 2009;64(4):337-43.

OBJECTIVE: To compare variations of plethysmographic wave amplitude ( $\Delta$ Ppleth) and to determine the percent difference between inspiratory and expiratory pulse pressure $(\Delta \mathrm{Pp})$ cutoff values for volume responsiveness in a homogenous population of postoperative cardiac surgery patients.

INTRODUCTION: Intra-thoracic pressure variations interfere with stroke volume variation. Pulse pressure variations through arterial lines during mechanical ventilation have been recommended for the estimation of fluid responsiveness. Pulse oximetry may offer a non-invasive plethysmographic method to evaluate pulse pressure; this may be useful for guiding fluid replacement.

METHODS: Controlled, prospective study in cardiac surgery patients under controlled ventilation. Simultaneous digital recordings of arterial pressure and plethysmographic waves were performed. $\Delta \mathrm{Pp}$, systolic pressure $(\Delta \mathrm{Ps}), \Delta \mathrm{P}$ pleth, and systolic component ( $\Delta$ Spleth) were calculated. A $\Delta \mathrm{Pp} \geq 13 \%$ identified fluid-responsive patients. Volume expansion was performed in responsive subjects. Systolic and amplitude components of pressure and plethysmographic waves were compared.

RESULTS: In 50 measurements from 43 patients, $\Delta \mathrm{Pp}$ was correlated with (Ppleth $(\mathrm{r}=0.90, \mathrm{p}<0.001),(\mathrm{Ps}(\mathrm{r}=0.90, \mathrm{p}<0.001)$, and (Spleth $(\mathrm{r}=0.73, \mathrm{p}<0.001)$. An aArea under ROC curve (AUC) identified the fluid responsiveness thresholds: (Ppleth of 11\% (AUC $=0.95 \pm 0.04),(\mathrm{Ps}$ of $8 \%(\mathrm{AUC}=0.93 \pm 0.05)$, and (Spleth of $32 \%(\mathrm{AUC}=0.82 \pm 0.07)$. A (Ppleth value $\geq 11 \%$ predicted $(\mathrm{Pp} \geq 13 \%$ with $100 \%$ specificity and $91 \%$ sensitivity. Volume expansion, performed in 20 patients, changed (Pp, (Ppleth, (Ps and (Spleth significantly $(\mathrm{p}<0.008)$.

CONCLUSIONS: $\triangle$ Ppleth is well correlated with $\triangle \mathrm{Pp}$ and constitutes a simple and non-invasive method for assessing fluid responsiveness in patients following cardiac surgery.

KEYWORDS: Volume replacement; Hemodynamics; Postoperative care; Cardiac function; Cardiac catheterization/intervention.

\section{INTRODUCTION}

Changes in intra-thoracic pressure interfere with venous return and cardiac output. Varying the arterial pressure

\footnotetext{
I Intensive Care Unit, Centro Hospitalar Unimed Joinville - Joinville/SC, Brazil.

II Intensive Care Unit, Hospital Israelita Albert Einstein - São Paulo/SP, Brazil.

III Department of Surgery, LIM 26, Faculdade de Medicina da Universidade de São Paulo - São Paulo/SP, Brazil.

Email: luizpoli@usp.br

Tel.: 55113061.7474

Received for publication on December 07, 2008

Accepted for publication on January 13, 2009
}

through arterial lines during mechanical ventilation has been recommended for testing cardiovascular responsiveness during volume replacement in critically ill patients. As arterial systolic pressure and plethysmographic systolic component variations ( $\Delta$ Spleth) could change similarly, $\Delta$ Spleth has been proposed as a useful tool for determining recruitable preload in the absence of invasive pressure monitoring. ${ }^{1}$

Arterial pulse pressure respiratory variation $(\Delta \mathrm{Pp})$ has greater specificity and sensitivity for detecting cardiovascular responsiveness to volume expansion than $\Delta \mathrm{Ps}$. Therefore, we originally demonstrated the usefulness of the respiratory variation of the plethysmographic waveform amplitude 
$(\Delta$ Ppleth) to test cardiovascular responsiveness after volume expansion in critically ill septic, hypovolemic, postoperative and cardiac patients. $^{2}$ Other studies have corroborated our findings, observing a strong relationship between $\Delta \mathrm{Pp}$ and $\Delta$ Ppleth in similar clinical settings. ${ }^{3,4}$

Although the aforementioned studies ${ }^{2-4}$ suggest that pulse oximetry may offer a noninvasive plethysmographic evaluation to guide fluid infusion, the correspondence between $\Delta \mathrm{P}$ pleth and $\Delta \mathrm{Pp}$ has not been evaluated after fluid challenge or by cardiovascular responsiveness through cardiac output measurement. Along these lines, some studies ${ }^{5,6}$ have compared $\Delta \mathrm{Pp}$ and $\Delta \mathrm{Ppleth}$ with effective cardiac response measured by thermodilution and echocardiography, respectively. These studies demonstrated that $\Delta$ Ppleth was useful for predicting fluid responsiveness, as were analogous indices derived from direct arterial blood pressure measurements. ${ }^{5,6}$ However, these studies also showed a clear discrepancy in $\Delta$ Ppleth threshold values for fluid responsiveness identification. ${ }^{5,6} \Delta$ Ppleth values oscillated between $9 \%$ and $15 \%$, and greater values were observed when septic patients predominated in the study populations..$^{2-6}$

Hence, in this study we compared $\Delta$ Ppleth and $\Delta \mathrm{Pp}$, and determined the $\Delta$ Ppleth cutoff value for volume responsiveness in a homogenous population of patients following cardiac surgery.

\section{METHODS}

This was a prospective, non-interventional study performed in a mixed intensive care unit of the Centro Hospitalar Unimed, Joinville, Brazil, from October 2003 to December 2004, after approval by the ethics committee. Written consent was obtained from each patient or next of kin. Forty-three consecutive adult postoperative cardiac surgery patients were enrolled.

Inclusion criteria: Patients were included if under mechanical ventilation and residual effects of sedation and muscle relaxation in a three hour window after cardiac surgery.

Exclusion criteria: Patients were excluded if presenting one or more of the following conditions: cardiac arrhythmias, spontaneous breathing movements, positive end-expiratory pressure (PEEP) higher than $8 \mathrm{~cm} \mathrm{H}_{2} \mathrm{O}$, tidal volume lower than $8 \mathrm{~mL} / \mathrm{kg}$ or higher than $10 \mathrm{~mL} / \mathrm{kg}$, auto-PEEP and/or bronchospasm. ${ }^{7}$ Patients without informed consent were not included.

\section{Interventions}

All patients were under controlled mode ventilation (Puritan Bennett 7200; Puritan Bennett, Carlsbad, Calif or
Newport Wave E200; Newport Beach, Calif), tidal volume between 8 and $10 \mathrm{~mL} / \mathrm{kg}$, inspiratory/expiratory ratio of 1:3, PEEP between 5 and $8 \mathrm{~cm} \mathrm{H}_{2} \mathrm{O}$, and $\mathrm{FiO}_{2}$ to maintain arterial oxygen saturation around $95 \%$. Additional sedative or muscle relaxant drugs were used at the discretion of the attending physician. All patients received an arterial line into the radial artery through a 20-gauge catheter (Abbocath, Becton Dickinson, Franklin Lakes, NJ). A double-lumen catheter (MAC; Multi-Lumen Access Catheter, Arrow International Inc, Bernville Road Reading, PA) was placed in the superior vena cava through the subclavian or internal jugular vein.

An oxymeter sensor was adapted to the indicator finger on the same side as the arterial catheterization. This oxymeter was a modular unit from a multi-parametric monitor (S/5; Datex-Ohmeda, Helsinki, Finland). Blood samples from central venous and arterial lines were obtained for blood gas analysis. All pressure transducers were referenced to mid-chest. Mechanical ventilator settings and vasoactive drug regimens were unchanged during the study protocol.

\section{Measurements}

Heart rate $(\mathrm{HR})$, respiratory rate (RR), central venous pressure (CVP), invasive mean arterial pressure (MAP), electrocardiography, plethysmographic waves of pulse oximetry, airway pressure, PEEP, and tidal volume were continuously monitored, and tracings were simultaneously registered. During a 60-second period, invasive arterial, pulse oximetry plethysmographic tracings, and time-pressure respiratory curves were simultaneously registered and transferred from a multi-parametric monitor (S/5; DatexOhmeda, Helsinki, Finland) to a personal computer (S/5 Collect; Datex-Ohmeda, Helsinki, Finland). During data acquisition, no spontaneous respiratory movements were present. Therefore, respiratory-induced changes in arterial and plethysmographic waves were due exclusively to the effects of mechanical ventilation on intrathoracic pressures.

The analysis of pressure and plethysmographic waveforms was performed off-line on a personal computer. We first identified arterial pressure respiratory variation and the systolic and diastolic pressures (Figure 1). Then, systolic pressure and pulse pressure during inspiratory $\left(\mathrm{Ps}_{\max }\right.$ and $\left.\mathrm{Pp}_{\max }\right)$ and expiratory $\left(\mathrm{Ps}_{\min }\right.$ and $\mathrm{Pp}_{\min }$ ) phases were identified in each respiratory cycle. Respiratory variations in systolic pressure $(\Delta \mathrm{Ps}$, in $\%)$ and pulse pressure $(\Delta \mathrm{Pp}$, in $\%)$ were calculated using the following formulae: ${ }^{7}$

$$
\begin{aligned}
& \Delta \mathbf{P s}(\%)=100 \times\left(\mathbf{P s}_{\max }-\mathbf{P s}_{\min }\right) /\left[\left(\mathbf{P s}_{\max }+\mathbf{P s}_{\min }\right) / 2\right] \\
& \Delta \mathbf{P p}(\%)=100 \times\left(\mathbf{P p}_{\max }-\mathbf{P p}_{\min }\right) /\left[\left(\mathbf{P p}_{\max }+\mathbf{P p}_{\min }\right) / 2\right]
\end{aligned}
$$




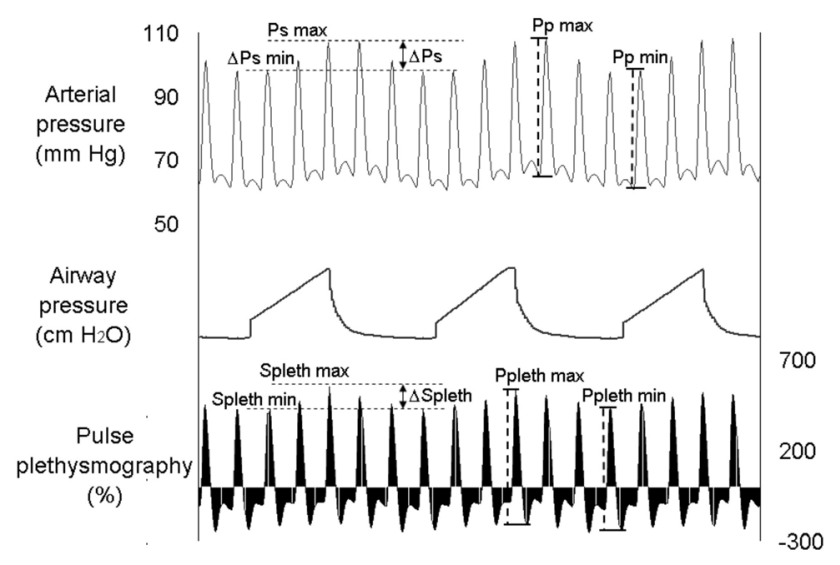

Figure 1 - Simultaneous fluctuations in arterial pressure (top) and plethysmographic (bottom) waveforms during mechanical ventilation. The systolic pressure and systolic plethysmographic component are maximal during inspiration and decline proportionally in expiration. Similarly, the arterial pulse pressure and plethysmographic amplitude are maximal during inspiration and minimal during expiration. $\mathrm{Ps}_{\max }$, maximal arterial systolic pressure; Spleth $_{\max }$, maximal plethysmographic waveform systolic component; $\mathrm{Ps}_{\min }$, minimal arterial systolic pressure; Spleth $_{\min }$, minimal plethysmographic waveform systolic component; $\mathrm{Pp}_{\max }$, maximal arterial pulse pressure; Ppleth ${ }_{\max }$, maximal plethysmographic waveform amplitude; $\mathrm{Pp}_{\text {min }}$, minimal arterial pulse pressure; Ppleth ${ }_{\text {min }}$, minimal plethysmographic waveform amplitude.

Plethysmographic waveforms were also evaluated using a similar methodology. Plethysmographic and arterial waveforms were simultaneously recorded and selected for analysis. The systolic component and the amplitude of the plethysmographic wave in the inspiratory phase (Spleth ${ }_{\text {max }}$ and $\Delta$ Ppleth $_{\max }$ ) and expiratory phase (Spleth ${ }_{\text {min }}$ and $\Delta$ Ppleth $_{\text {min }}$ ) were determined. Respiratory variations in the systolic plethysmographic component $(\Delta$ Spleth, in $\%)$ and respiratory variations in plethysmographic amplitude ( $\Delta$ Ppleth, in \%) were calculated using the following formulae:

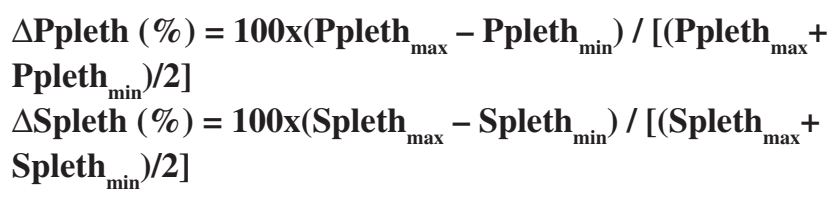

Each unit of the plethysmographic scale displayed on the secondary y axis depicted in figure 1 corresponds to the percent relationship between the pulse sign and the static sign detected by pulse oximeter.

$\Delta \mathrm{Ps}, \Delta \mathrm{Pp}, \Delta \mathrm{P}$ pleth and $\Delta$ Spleth were determined as the average of three respiratory cycles over one minute. From these measurements, a correlation coefficient was calculated, and a cutoff value for $\Delta$ Ppleth and $\Delta$ Spleth was identified.

\section{Fluid responsiveness}

We have defined fluid responsiveness as a $\Delta \mathrm{Pp} \geq 13 \%$ in a previous study, ${ }^{7}$ and non-fluid responsiveness as a $\Delta \mathrm{Pp}<13 \%$. We did not measure cardiac output or systolic volume.

\section{Fluid challenge}

According to clinical judgment, patients were challenged with a normal saline infusion, ranging from 500 to 1000 $\mathrm{mL}$. HR, MAP, CVP, $\Delta \mathrm{Ps}, \Delta \mathrm{Pp}, \Delta$ Ppleth and $\Delta$ Spleth were analyzed before and after fluid challenge.

\section{Clinical characteristics}

Eventual influences of clinical variables on pressure and plethysmographic traces relationship were examined. Clinical variables were: age, time on extra-corporeal circulation, temperature, MAP, CVP, hematocrit, $\mathrm{ScvO}_{2}$, $\mathrm{PaCO}_{2}$ and plethysmographic sign.

\section{Reproducibility}

The agreement between two independent observers was evaluated to test the reproducibility of plethysmographic wave respiratory variation in volemic status determination.

\section{Statistical analysis}

NCSS Statistical Software 2000 \& PASS 2000: Power Analysis \& Sample Size (NCSS Kaysville, Utah) was used for statistical analysis. Data were compared using Student's $t$ test for continuous variables. Ordinal data or non-normally distributed continuous data were compared by the nonparametric Wilcoxon rank sum test for paired observations. Correlations were determined using the Spearman test. The agreement between $\Delta \mathrm{Pp}$ and $\Delta \mathrm{Ppleth}$ for potential responsiveness was evaluated by Cohen kappa index. Receiver operating characteristic curves were generated for $\Delta \mathrm{Ps}, \Delta \mathrm{P}$ pleth, $\Delta$ Spleth (in \%) and CVP (in $\mathrm{mmHg}$ ) to identify the threshold values between fluid responders and non-responders. We performed a stepwise multiple regression to identify eventual influences of clinical variables on the pressure and plethysmographic trace relationship. The concordance between independent observers was evaluated by Bland-Altmann analysis, Spearman's correlation test and the Cohen kappa index. Results are expressed as mean $\pm \mathrm{SD}$. All tests were twotailed, and $P<0.05$ was considered statistically significant.

\section{RESULTS}

Fifty simultaneous arterial pressure and pulse plethysmo- 
graphic traces were obtained from 43 postoperative cardiac surgery patients. Table 1 shows demographic, hemodynamic, and ventilatory data.

The linear correlation between $\Delta \mathrm{Pp}$ and $\Delta \mathrm{Ppleth}(r=$ 0.90, $P<0.001)$ is depicted in Figure 2. Other correlations were also observed: $\Delta \mathrm{Pp}$ and $\Delta \mathrm{Ps}(\mathrm{r}=0.90, P<0.001)$, $\Delta \mathrm{Pp}$ and $\Delta$ Spleth $(\mathrm{r}=0.73, P<0.001), \Delta \mathrm{Ps}$ and (Spleth $(\mathrm{r}$ $=0.61, \mathrm{p} \mathrm{P}<0.001)$ and (Ppleth and (Spleth $(\mathrm{r}=0.83, \mathrm{p} \mathrm{P}$ $<0.001)$.

We determined $11 \%$ as the $\Delta \mathrm{Pp}$ cutoff value to discriminate PFR and NPFR, with a sensitivity of $93 \%$, a specificity of $95 \%$, and an area under the ROC curve (AUC) of $0.95 \pm 0.04$. The $\Delta$ Ps threshold value was $8 \%$, with a

Table 1 - Patients characteristics $(n=43)$

\begin{tabular}{|c|c|}
\hline \multicolumn{2}{|l|}{ Gender } \\
\hline Male & 28 \\
\hline Female & 15 \\
\hline Age, years & $61 \pm 9$ \\
\hline Weight, kg & $72 \pm 10$ \\
\hline APACHE II score & $9.2 \pm 4.7$ \\
\hline \multicolumn{2}{|l|}{ Surgery } \\
\hline Coronary graft & 36 \\
\hline \multicolumn{2}{|l|}{ Valvular surgery } \\
\hline Aortic & 5 \\
\hline Mitral & 2 \\
\hline \multicolumn{2}{|l|}{ Hemodynamics } \\
\hline Heart rate, bpm & $92 \pm 19$ \\
\hline MAP, mm Hg & $82 \pm 11$ \\
\hline $\mathrm{ScvO}_{2}, \%$ & $56 \pm 9.5$ \\
\hline CVP, mm Hg & $7.3 \pm 3.5$ \\
\hline$\Delta \mathrm{Pp}, \%$ & $17 \pm 9$ \\
\hline$\Delta \mathrm{Ps}, \%$ & $10 \pm 4.1$ \\
\hline$\Delta$ Ppleth, $\%$ & $15 \pm 11$ \\
\hline$\Delta$ Spleth, $\%$ & $46 \pm 32$ \\
\hline \multicolumn{2}{|c|}{ Mechanical ventilatory settings } \\
\hline Tidal volume, $\mathrm{mL} / \mathrm{kg}$ & $9 \pm 1$ \\
\hline Peak pressure, $\mathrm{cm} \mathrm{H}_{2} \mathrm{O}$ & $30 \pm 3$ \\
\hline Respiratory rate, rpm & $14 \pm 2$ \\
\hline PEEP, $\mathrm{cm} \mathrm{H}_{2} \mathrm{O}$ & $5 \pm 1$ \\
\hline $\mathrm{PaCO}_{2}, \mathrm{~mm} \mathrm{Hg}$ & $33 \pm 6$ \\
\hline $\mathrm{SaO}_{2}, \%$ & $95 \pm 2$ \\
\hline
\end{tabular}

APACHE II, Acute Physiology and Chronic Health Evaluation II; CVP, central venous pressure; $\Delta \mathrm{Pp}$, pulse pressure respiratory variation; $\Delta \mathrm{Ppleth}$, respiratory variation of plethysmographic wave amplitude; $\Delta \mathrm{Ps}$, systolic pressure respiratory variation; $\Delta$ Spleth, systolic plethysmographic respiratory variation; MAP, mean arterial pressure; PEEP, positive end expiratory pressure; $\mathrm{SaO}_{2}$, arterial oxygen saturation; $\mathrm{ScvO}_{2}$, central venous oxygen saturation.

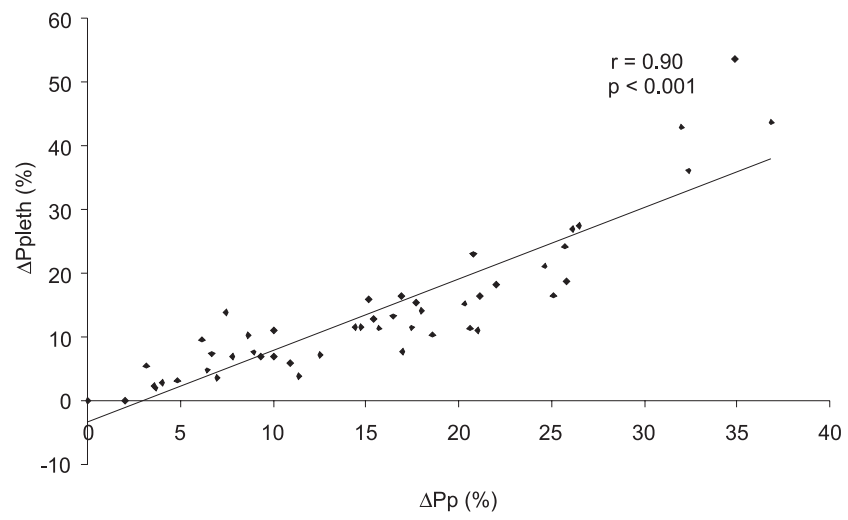

Figure 2 - Global correlation between $\Delta \mathrm{Pp}$ and $\Delta \mathrm{Ppleth}$ observed in fifty measurements performed in the 43 patients. $\Delta \mathrm{Pp}(\%)$, pulse pressure respiratory variation; $\Delta$ Ppleth (\%), respiratory variation of plethysmographic wave amplitude.

sensitivity of $95 \%$, a specificity of $91 \%$ and an AUC of 0.93 \pm 0.05 . The $\Delta$ Spleth cutoff was $32 \%$, with a sensitivity of $74 \%$, a specificity of $91 \%$ and an AUC of $0.82 \pm 0.07$. Using a mean CVP of $8 \mathrm{~mm} \mathrm{Hg}$ as the best cutoff value, we found an AUC of $0.59 \pm 0.1$, a sensitivity of $74 \%$ and a specificity of $45 \%$ (Figure 3). There was good agreement between $\Delta \mathrm{Pp}$ and $\Delta$ Ppleth as shown by kappa test of $0.88, P<0.001$ for PFR identification (Figure 4). We evaluated this concordance using the kappa index to compare $\Delta \mathrm{Pp}$ and $\Delta \mathrm{Ps}$ (kappa $=0.85, P<0.001), \Delta$ Pp and $\Delta$ Spleth (kappa $=0.60, P<$ $0.001), \Delta \mathrm{Pp}$ and CVP (kappa $=0.02, P<0.001)$.

In 20 patients given fluid challenge, $\Delta \mathrm{Pp}$ and $\Delta$ Ppleth decreased after fluid replacement, from $21 \% \pm 9 \%$ to $8 \%$ $\pm 5 \%(P<0.001)$ and from $19 \% \pm 10 \%$ to $5 \% \pm 4 \%(P<$ 0.001 ), respectively (Figure 5$). \Delta \mathrm{Ps}$ and $\Delta$ Spleth decreased from $12.3 \% \pm 3.5 \%$ to $7 \% \pm 2 \%(P<0.001)$ and $66 \% \pm 42 \%$

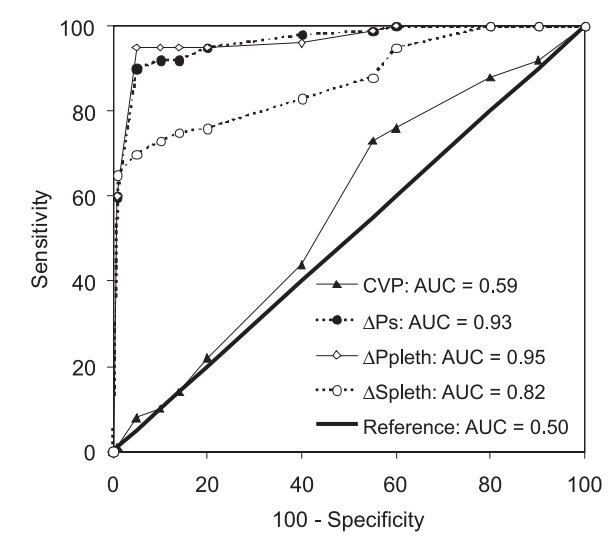

Figure 3 - ROC curves comparing the ability of $\triangle \mathrm{Ppleth}, \triangle \mathrm{Spleth}$ and $\mathrm{CVP}$ to reproduce $\Delta \mathrm{Pp}$ in discrimination of potential fluid responders $(\Delta \mathrm{Pp} \geq$ $13 \%)$ and non-potential fluid responders $(\Delta \mathrm{Pp}<13 \%)$. AUC, area under ROC curve; CVP, central venous pressure; $\Delta \mathrm{Pp}(\%)$,pulse pressure respiratory variation; $\Delta \mathrm{Ppleth}(\%)$, respiratory variation of plethysmographic wave amplitude; $\Delta \mathrm{Ps}(\%)$, systolic pressure respiratory variation; $\Delta$ Spleth $(\%)$, plethysmographic systolic component variation; ROC, receiver operating characteristic. 


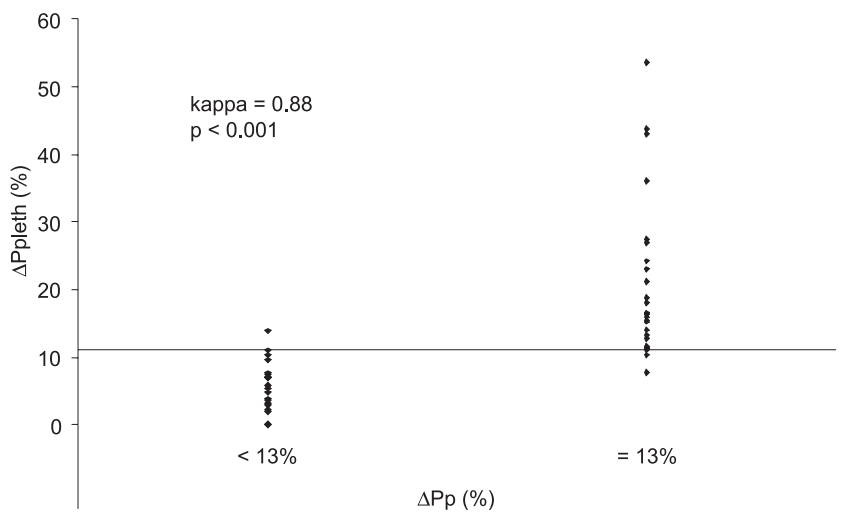

Figure 4 - Good agreement between $\Delta \mathrm{Pp}$ and $\Delta$ Ppleth. A $11 \% \Delta$ Ppleth cutoff value discriminated non-potential fluid responders $(\Delta \mathrm{Pp}<13 \%)$ and potential fluid responders $(\Delta \mathrm{Pp} \geq 13 \%) . \Delta \mathrm{Pp}(\%)$, pulse pressure respiratory variation; $\Delta$ Ppleth (\%), respiratory variation of plethysmographic wave amplitude.

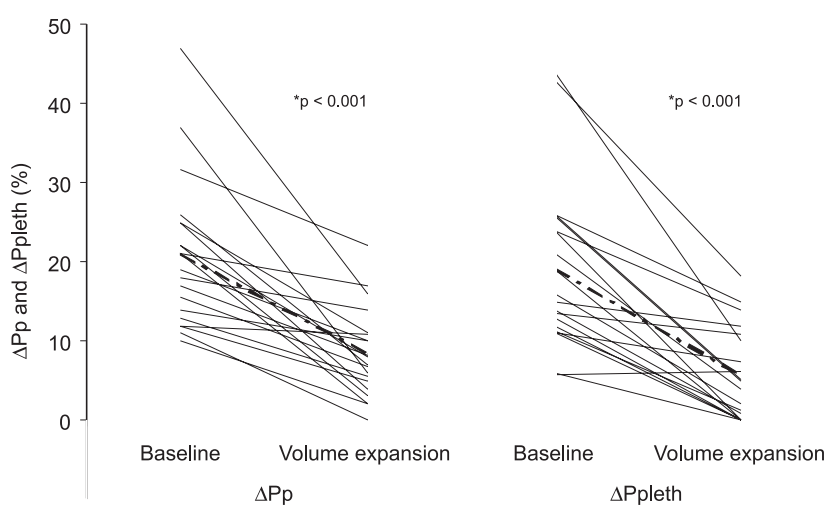

Figure 5 - Individual responses to volume expansion on $\Delta \mathrm{Pp}$ and $\Delta \mathrm{Ppleth}$ $(\%)$ in 20 patients. The dashed line shows mean decline of the parameters. *Wilcoxon rank sum test. $\Delta \mathrm{Pp}(\%)$, pulse pressure respiratory variation; $\Delta$ Ppleth (\%), respiratory variation of plethysmographic wave amplitude.

to $36 \% \pm 24 \%(P<0.008)$, respectively. Static parameters did not change after fluid infusion. CVP varied from $8 \pm 3.5$ to $9 \pm 5(P=0.08), \mathrm{HR}$ from $104 \pm 26$ to $102 \pm 25(P=0.3)$ and MAP from $71 \pm 13$ to $77 \pm 15(P=0.4)$. We determined the correlations between $\Delta \mathrm{Ps}$ and $\Delta$ Spleth before $(\mathrm{r}=0.85$, $P=0.02)$ and after fluid expansion $(\mathrm{r}=0.23, P=0.55)$, and between $\Delta \mathrm{Pp}$ and $\Delta$ Ppleth before $(\mathrm{r}=0.73, P=0.03)$ and after infusion ( $\mathrm{r}=0.84, P=0.004)$.

PFR and NPFR clinical parameters are shown in Table 2. Age, Acute Physiology and Chronic Health Evaluation II (APACHE II) score, $\mathrm{PaCO}_{2}$, central venous oxygen saturation $\left(\mathrm{ScvO}_{2}\right)$, heart rate, mean arterial pressure, and CVP were similar in both groups, while $\Delta \mathrm{Pp}, \Delta \mathrm{Ps}, \Delta \mathrm{Ppleth}$ and $\Delta$ Spleth were all statistically different $(P<0.001)$.

Individual clinical characteristics did not influence the dynamic variables. Using a stepwise multiple regression we found that extracorporeal circulation time showed a significant positive correlation with $\Delta \mathrm{Pp}$ and $\Delta \mathrm{Ppleth}(P$ $<0.001)$, but not with $\Delta \mathrm{Ps}$ or $\Delta$ Spleth $(P=0.33)$. There
Table 2 - Clinical parameters according volume status

\begin{tabular}{lccc}
\hline & $\begin{array}{c}\Delta \mathrm{Pp}<13 \% \\
(\mathrm{n}=17)\end{array}$ & $\begin{array}{c}\Delta \mathrm{Pp} \geq 13 \% \\
(\mathrm{n}=26)\end{array}$ & $P^{\mathrm{a}}$ \\
\hline Age, years & $60 \pm 10$ & $62 \pm 8$ & 0.45 \\
APACHE II & $9 \pm 3$ & $10 \pm 5$ & 0.57 \\
$\mathrm{PaCO}_{2}, \mathrm{~mm} \mathrm{Hg}$ & $34 \pm 5$ & $33 \pm 7$ & 0.85 \\
$\mathrm{ScvO}_{2}, \%$ & $54 \pm 10$ & $58 \pm 8.5$ & 0.59 \\
$\mathrm{HR}, \mathrm{bpm}$ & $86 \pm 15$ & $97 \pm 20$ & 0.11 \\
MAP, mm Hg & $82 \pm 11$ & $82 \pm 12$ & 0.51 \\
CVP, mm Hg & $7 \pm 3.5$ & $7 \pm 3.4$ & 0.51 \\
$\Delta$ Pp, \% & $7 \pm 2.8$ & $22 \pm 6$ & $<0.001$ \\
$\Delta$ Ps, \% & $7 \pm 2.4$ & $12 \pm 4.5$ & $<0.001$ \\
$\Delta$ Ppleth, \% & $6 \pm 2.5$ & $21 \pm 11$ & $<0.001$ \\
$\Delta$ Spleth, \% & $23 \pm 8$ & $58 \pm 35$ & $<0.001$ \\
\hline
\end{tabular}

APACHE II, Acute Physiology and Chronic Health Evaluation II; CVP, central venous pressure; $\Delta \mathrm{Pp}$, pulse pressure respiratory variation; $\Delta \mathrm{Ppleth}$, respiratory variation of plethysmographic wave amplitude; $\Delta \mathrm{Ps}$, systolic pressure respiratory variation; $\Delta$ Spleth, systolic plethysmographic respiratory variation; $\mathrm{HR}$, heart rate; MAP, mean arterial pressure; $\mathrm{SaO}_{2}$, arterial oxygen saturation; $\mathrm{ScvO}_{2}$, central venous oxygen saturation. ${ }^{\mathrm{a} t}$-Student test.

were no significant differences in the correlation between the behavior of $\Delta$ Ppleth and (Spleth in relation to (Pp in 11 patients $(\mathrm{P}=0.1)$ receiving vasopressors (noradrenaline, $\mathrm{N}=3,0.5 \pm 0.15 \mathrm{mcg} / \mathrm{kg} / \mathrm{min}$ or dopamine, $\mathrm{N}=8,4.6 \pm 2$ $\mathrm{mcg} / \mathrm{kg} / \mathrm{min})$, in 5 patients $(\mathrm{P}=0.32)$ receiving dobutamine $(6.6 \pm 2.43 \mathrm{mcg} / \mathrm{kg} / \mathrm{min})$, or in 6 patients $(\mathrm{P}=0.34)$ receiving nitroglycerin $(0.4 \pm 0.2 \mathrm{mcg} / \mathrm{kg} / \mathrm{min})$.

The inter-observer $\Delta \mathrm{Pp}$ reproducibility analysis showed a good correlation $(r=0.92, P<0.001)$. The mean difference between observers was $1.0 \pm 3.2$, with a superior limit of 7.4 and an inferior limit of -5.2. The agreement between observers for discrimination of PFR from NPFR was also good (kappa $=0.92 ; P<0.001)$. A similar agreement was found for $\Delta$ Ppleth inter-observer analysis $(r=0.94, P<$ 0.001 ; mean difference $=1.0 \pm 3.3$, superior limit $=7.2$, inferior limit $=-5.7$; and kappa $=0.85 ; P<0.001)$.

\section{DISCUSSION}

We were able to demonstrate a correlation between pulse oximetry plethysmographic wave amplitude and arterial pulse pressure variations induced by respiratory cycles in cardiac surgery patients. Therefore, the non-invasive plethysmographic wave amplitude respiratory variation measurement could potentially be used to detect patients who could benefit from additional fluid loading.

Arterial pressure variation has long been considered an accurate surrogate marker of fluid responsiveness. ${ }^{7-15}$ The role of $\Delta \mathrm{Ps}$ as a marker of volemic status was initially 
suggested by Perel et al. in $1987 .{ }^{13}$ More recently, Michard et al. demonstrated the usefulness of the $\Delta \mathrm{Pp}$ measurement in showing fluid responsiveness in septic patients. ${ }^{7}$ Although $\Delta \mathrm{Pp}$ measurement has been considered a minimally invasive technique, it is time-consuming and associated with rare but potentially harmful complications secondary to arterial cannulation, including hematoma, infection and thrombosis. ${ }^{1}$ In contrast, since the pulse oximetry plethysmographic signal resembles the peripheral arterial pressure waveform and the degree of respiratory variation in the pulse oximetry wave is close to the degree of respiratory arterial pulse pressure variation, this measurement could be a useful and noninvasive alternative to $\Delta \mathrm{Pp} .{ }^{16}$

The recognition of blood flow by the pulse oximetry plethysmographic sensor results from light emission by diodes (light emitting diode - LED). The detected pulsatility results from attenuation of the cyclic light energy transmitted to or reflected by the tissues where the sensor is applied. The light absorption by hemoglobin within the arteries and arterioles increases during the systole and decreases during diastole, due to the pulsatile variation of the blood volume and erythrocyte mass under the sensor. ${ }^{16-18}$ The analysis of arterial and/or pulse plethysmographic wave tracings has been proposed as a tool to evaluate the relationship between plethysmographic wave behavior and the presence of paradoxical pulse. ${ }^{19,20}$ Dorlas et al. ${ }^{21}$ and Partridge et al. ${ }^{22}$ have shown correlations in the variation of pulse plethysmographic wave with preload and systolic volume. In addition, Shamir et al. ${ }^{1}$ observed a good correlation between $\Delta \mathrm{Ps}$ and $\Delta$ Spleth after withdrawal of $10 \%$ of blood volume and reinfusion. However, we must consider that while $\Delta \mathrm{Ps}$ results from systolic volume variation and the impact of inspiratory pleural pressure on the aorta, $\Delta \mathrm{Pp}$ reflects only systolic volume variation. Hence, $\Delta \mathrm{Ps}$ is more sensitive but less specific than $\Delta \mathrm{Pp}$ in detecting fluid load responsiveness, explaining the lower performance of (Spleth in comparison to (Ppleth). ${ }^{7}$

The correlation between (Ppleth and (Pp was pioneered by our group in 2004.2 Later, Cannesson et al., ${ }^{3}$ Natalini et al., ${ }^{4}$ and Feissel et al. ${ }^{6}$ reported similar correlation coefficients to those we found. In the present study, interobserver reproducibility analyses revealed an excellent linear correlation and a strong agreement between independent observers, highlighting the reproducibility of (Ppleth. Second, among the several clinical variables that could interfere with the proposed comparisons, the extracorporeal circulation time was the only one related to the magnitude of both (Pp and (Ppleth. It is well known that extracorporeal circulation elicits an inflammatory response, reduces vascular tonus and increases endothelial permeability. These factors could alter (Pp and (Ppleth behavior in the same way). ${ }^{23,24}$
Third, we evaluated $\Delta \mathrm{Ppleth}, \Delta \mathrm{Spleth}, \Delta \mathrm{Pp}$, and $\Delta \mathrm{Ps}$ in a homogeneous group of cardiac surgery patients, finding a $\Delta$ Ppleth cutoff value of $11 \%$. Previous studies enrolling a more heterogeneous population reported different cutoff values for $\Delta$ Ppleth, ranging from $9 \%$ and $15 \%$, for a considered gold standard $\Delta \mathrm{Pp}$ value of $13 \%$. $^{2-6}$ Natalini et al. ${ }^{4}$ observed a threshold of $9 \%$ in a general population. In a subsequent study in which more than $70 \%$ of patients were septic, a $\Delta$ Ppleth cutoff value of $15 \%$ was reported by addressing cardiovascular responsiveness through thermodilution. ${ }^{5}$ We reported a $\Delta$ Ppleth cutoff value of $10 \%$ in a study in which $15 \%$ of patients were septic. ${ }^{2}$ Recently, Feissel et al. showed a (Ppleth cutoff of $14 \%$ using echocardiographic cardiac output measurements in septic patients. 6 Wyffels et al. included only cardiac surgery patients and found a cutoff value of $11 \%$, as reported here. 25 Apparently, greater values of (Ppleth allow the identification of cardiovascular responsiveness among septic patients, who frequently present widespread vasodilation. In agreement with Shamir et al,1 we consider that the high sensitivity and variability of the plethysmographic signals are likely caused by humoral and neurogenic factors.1,18,19 The (Ppleth amplification in relation to (Pp could be explained by the humoral response increasing the arteriolar compliance during arterial pressure measurement.

Fourth, there were concomitant changes in (Ppleth, (Spleth, (Pp and (Ps before and after volume challenge. However, there are some limitations to this aspect of the study, including the lack of left ventricular function analysis and the fact that we did not measure cardiac output directly in order to discriminate fluid responders from non-responders, since this is not routinely done in our hospital. We therefore classified our patients as "potential responders" ((Pp $\geq 13 \%)$ and "potential non-responders" ( $(\mathrm{Pp}<13 \%)$, based on studies in septic patients and cardiac surgery patients.9,26,27 In a recent publication, Natalini et al. 5 performed thirty-two fluid challenges in 22 hypotensive patients who were also monitored with a pulmonary artery catheter, showing that (Ppleth could predict cardiac output increase by administering fluid).

It must be noted the available pulse plethysmographs have limitations such as the lack of scales in the screen, and have fixed mechanisms to attenuate or increase the signal according to intensity. ${ }^{16-19}$ We believe that new pulse oximetry devices could be easily adapted or developed if this concept is widely accepted. Other limitations have also been attributed to dynamic indicators of cardiovascular responsiveness, including movement artifacts, peripheral vasoconstriction and cutaneous pigments. ${ }^{16,28}$ Although our findings were obtained under highly controlled conditions, mechanical ventilation and pulse plethysmography are 
frequently required for critically ill or complex surgical patients.

We conclude that $\Delta$ Ppleth of $11 \%$ or more accurately identifies respiratory pulse pressure variations greater than
$13 \%$ in post-cardiac surgery patients under mechanical ventilation. In this homogeneous population, $\Delta$ Ppleth has the potential to determine the response to fluid challenge in critically ill patients in the absence of an arterial line.

\section{REFERENCES}

1. Shamir M, Eidelman LA, Floman Y, Kaplan L, Pizov R. Pulse oximetry plethysmographic waveform during changes in blood volume. Br J Anaesth. 1999;82:178-81.

2. Poli de Figueiredo LF, Silva E, Rocha e Silva M, Westphal GA, Caldeira Filho M. Pulse oximetry wave respiratory variations for the assessment of volume status in patients under mechanical ventilation. Crit Care Med. 2004;32:A96.

3. Cannesson M, Besnard C, Durand PG, Bohé J, Didier Jacques. Relation between respiratory variations in pulse oximetry plehysmographic waveform amplitude and arterial pulse pressure in ventilated patients. Critical Care. 2005;9:562-8

4. Natalini G, Rosano A, Franceschetti ME, Facchetti P, Bernardini A. Variations in arterial blood pressure and photoplethysmography during mechanical ventilation. Anesth Analg. 2006;103:1182-8 .

5. Natalini G, Rosano A, Taranto M, Faggian B, Vitorielli E, Bernardini A. Arterial versus plethysmographic dynamic indices to test responsiveness for testing fluid administration in hypotensive patients: A clinical trial. Anesth Analg. 2006;103:1478-84

6. Feissel M, Teboul JL, Merlani P, Badie J, Faller JP, Bendjelid K. Plethysmographic dynamic indices predict fluid responsiveness in septic ventilated patients. Intensive Care Med. 2007;33:993-9.

7. Michard F, Boussat S, Chemla D, Anguel N, Mercat A, Lecarpentier $\mathrm{Y}$, et al. Relation between respiratory changes in arterial pulse pressure and fluid responsiveness in septic patients with acute circulatory failure. Am J Respir Crit Care Med. 2000;162:134-8.

8. Auler JO Jr, Galas F, Hajjar L, Santos L, Carvalho T, Michard F. Online monitoring of pulse pressure variation to guide fluid therapy after cardiac surgery. Anesth Analg.2008;106:1201-6.

9. Michard F, Teboul JL. Predicting fluid responsiveness in ICU patients. A critical analysis of the evidence. Chest. 2002;121:2000-8.

10. Martin G. Fluid balance and colloid osmotic pressure in acute respiratory failure: emerging clinical evidence. Crit Care. 2000;4(Suppl 2):21-5.

11. Pinsky MR. Assessment of indices of preload and volume responsiveness. Curr Opin Crit Care. 2005;11:235-9.

12. Diebel L, Wilson RF, Heins J, Larky H, Warsow K, Wilson S. End diastolic volume versus pulmonary artery wedge pressure in evaluating cardiac preload in trauma patients. J Trauma. 1994;37:950-5.

13. Perel A, Pizov R, Cotev S. Systolic blood pressure variation is a sensitive indicator of hypovolemia in ventilated dogs subjected to graded hemorrhage. Anesthesiology. 1987;67:498-502.

14. Lopes MR, Auler JO Jr, Michard F. Volume management in critically ill patients: New insights. Clinics. 2006;61:345-50.
15. Westphal G, Garrido Adel P, de Almeida DP, Rocha e Silva M, Poli-deFigueiredo LF. Pulse pressure respiratory variation as an early marker of cardiac output fall in experimental hemorrhagic shock. Artif Organs. 2007;31:284-9.

16. Moyle JTB. Principles and practice series: Pulse oximetry. London: BMJ Books; 1998.

17. Jubran A. Pulse Oximetry. Crit Care. 1999;3: R11-R17.

18. Golparvar M, Naddafnia H, Saghaei M. Evaluating the relationship between arterial blood pressure changes and indices of pulse oximetric plethysmography. Anesth Analg. 2002;95:1686-90.

19. Hartert TV, Wheeler AP, Heller JR. Use of pulse oximetry to recognize of airflow obstruction in obstructive airway disease.Correlation with pulsus paradoxus. Chest. 1999;115:475-81.

20. Frey B, Butt W. Pulse oximetry for assessment of pulsus paradoxus: a clinical study in children. Intensive Care Med. 1998;24:242-6.

21. Dorlas JC, Nijboer JA: Photo-electric plethysmography as a monitoring device in anaesthesia. Application and interpretation. Br J Anaesth 1985;57:524-30.

22. Partridge BL. Use of pulse oximetry as a non invasive indicator of intravascular volume status. J Clin Monit 1987;3:263-8.

23. Laffey JG, Boylan JF, Cheng DC. The Systemic Inflammatory Response to Cardiac Surgery. Implications for the Anesthesiologist. Anesthesiology. 2002. 97(1):215-52.

24. Murphy GJ, Angelini GD. Side effects of cardiopulmonary bypass: What is the reality? J Card Surg. 2004;19:481-8.

25. Wyffels PA, Durnez PJ, Helderweirt J, Stockman WM, De Kegel D. Ventilation-induced plethysmographic variations predict fluid responsiveness in ventilated postoperative cardiac surgery patients. Anesth Analg. 2007;105:448-52.

26. Hofer CK, Müller SM, Furrer L, Klaghofer R, Genoni M, Zollinger A. Stroke volume and pulse pressure variation for prediction of fluid responsiveness in patients undergoing off-pump coronary artery bypass Grafting. Chest. 2005;128:848-54.

27. Kramer A, Zygun D, Hawes H, Easton P, Ferland A. Pulse pressure variation predicts fluid responsiveness following coronary artery bypass surgery. Chest. 2004;126:1563-8.

28. Pinsky MR. Assessment of indices of preload and volume responsiveness. Curr Opin Crit Care. 2005;11:235-9. 
